\title{
An audit of some processing effects in aggregated occurrence records
}

\author{
Robert Mesibov' \\ I West Ulverstone, Tasmania, Australia 7315 \\ Corresponding author: Robert Mesibov (robert.mesibov@gmail.com)
}

Academic editor: P. Stoev | Received 4 March 2018 | Accepted 15 April 2018 | Published 20 April 2018

http://zoobank.org/B58F9924-294F-4B4B-88C7-B9C366C20635

Citation: Mesibov R (2018) An audit of some processing effects in aggregated occurrence records. ZooKeys 751: 129-146. https://doi.org/10.3897/zookeys.751.24791

\begin{abstract}
A total of ca 800,000 occurrence records from the Australian Museum (AM), Museums Victoria (MV) and the New Zealand Arthropod Collection (NZAC) were audited for changes in selected Darwin Core fields after processing by the Atlas of Living Australia (ALA; for AM and MV records) and the Global Biodiversity Information Facility (GBIF; for AM, MV and NZAC records). Formal taxon names in the genus- and species-groups were changed in $13-21 \%$ of AM and MV records, depending on dataset and aggregator. There was little agreement between the two aggregators on processed names, with names changed in two to three times as many records by one aggregator alone compared to records with names changed by both aggregators. The type status of specimen records did not change with name changes, resulting in confusion as to the name with which a type was associated. Data losses of up to $100 \%$ were found after processing in some fields, apparently due to programming errors. The taxonomic usefulness of occurrence records could be improved if aggregators included both original and the processed taxonomic data items for each record. It is recommended that end-users check original and processed records for data loss and name replacements after processing by aggregators.
\end{abstract}

\section{Keywords}

Atlas of Living Australia, GBIF, occurrence records, data errors 


\section{Introduction}

Neither the Atlas of Living Australia (ALA) nor the Global Biodiversity Information Facility (GBIF) simply republishes the occurrence records it receives from data providers. Each aggregator processes incoming data in an effort to improve data quality. The processing works by adding, deleting or modifying data items, or by adding "assertions" (ALA) or "flags" (GBIF) to records that contain items identified as incorrect, incomplete, suspect or otherwise invalid.

Processing of this kind can be beneficial when data errors are corrected or flagged. Processing is not helpful when valid data items are lost and when added or modified data items are incorrect. To investigate some of the effects of processing I audited ca 345,000 occurrence records from the Australian Museum (AM) and ca 355,000 occurrence records from Museums Victoria (MV), in each case as the records appear in both ALA and GBIF. I was mainly interested in the changes made by the aggregators to taxon names, but I also checked for data losses in selected non-taxonomic fields, and for the latter purpose I examined ca 100,000 occurrence records in GBIF from the New Zealand Arthropod Collection (NZAC).

As reported below, some processing operations significantly downgraded rather than upgraded data quality, and changes in taxon names varied substantially between aggregators.

New processing routines are occasionally introduced by ALA and GBIF, and old ones improved over time (e.g., see the "Issues" section of the ALA "biocache-store" GitHub site, https://github.com/AtlasOfLivingAustralia/biocache-store). For this reason the results presented here should be seen as "date-stamped" early 2018, when I downloaded the sample data.

\section{Methods}

\section{Data sources}

From the ALA website I downloaded the "Australian Museum Malacology Collection" and "Museums Victoria provider for OZCAM" datasets. I chose Darwin Core (Wieczorek et al. 2012) downloads in TSV format with all fields in the record-class terms, occurrence, organism, event, location, identification, and taxon categories. From the ALA-MV dataset I selected all records with the collectionCode "Entomology". Each ALA table contains both original and processed data.

From the GBIF website I downloaded Darwin Core archives containing both original (verbatim.txt) and processed (occurrence.txt) record tables for "Australian Museum provider for OZCAM" and "Museums Victoria provider for OZCAM". From the AM dataset I selected original and processed records with the collectionCode "Malacology" and from the MV dataset the original and processed collectionCode "Entomology" records. 
I also downloaded from GBIF ca 100,000 original and processed records from the New Zealand Arthropod Collection (NZAC). The NZAC dataset had internal data problems that prevented me from auditing its taxonomic content effectively, but some GBIF processing effects on non-taxonomic NZAC data are noted in Results.

Download or data citations as recommended by ALA and GBIF are as follows:

AM Malacology from ALA

Atlas of Living Australia occurrence download at https://biocache.ala.org.au/occurrences/search?\&q=collection_uid\%3Aco1 14 accessed on Wed Feb 14 18:44:13 AEDT 2018

AM from GBIF

Australian Museum (2017). Australian Museum provider for OZCAM. Occurrence Dataset https://doi.org/10.15468/e7susi accessed via GBIF.org on 2018-02-14

MV from ALA

Atlas of Living Australia occurrence download at https://biocache.ala.org.au/ occurrences/search?\&q=data_resource_uid\%3Adr342 accessed on Wed Jan 31 06:42:40 AEDT 2018

MV from GBIF

Museums Victoria (2017). Museums Victoria provider for OZCAM. Occurrence Dataset https://doi.org/10.15468/lp1ctu accessed via GBIF.org on 2018-01-30

\section{NZAC from GBIF}

Wilton A (2018). New Zealand Arthropod Collection (NZAC). Version 1.67. Landcare Research. Occurrence Dataset https://doi.org/10.15468/lrgzz9 accessed via GBIF.org on 2018-01-08

After finding disagreements between ALA downloads and the ALA website (see Results and Discussion), I downloaded two additional record sets for checking, rather than auditing:

AM from ALA (standard download)

Atlas of Living Australia occurrence download at https://biocache.ala.org.au/occurrences/search?\&q=collection_uid\%3Aco1 14 accessed on Mon Feb 19 10:40:22 AEDT 2018

MV from ALA (standard download)

Atlas of Living Australia occurrence download at https://biocache.ala.org.au/occurrences/search?\&q=collection_uid\%3Aco39 accessed on Mon Feb 19 10:39:30 AEDT 2018 


\section{Data auditing and preparation}

I audited the records tables on the command line with BASH and GNU text-processing tools and GNU AWK 4 (Robbins 2018). The eight original working tables (AM and MV data from ALA; AM, MV and NZAC data from GBIF) have been archived in Zenodo (https://doi.org/10.5281/zenodo.1217733; version 2 uploaded 2018-04-13).

For convenience in cross-checking the results of ALA and GBIF processing, I reduced the AM and MV datasets to records with a catalogNumber in common between ALA and GBIF, i.e. 345,944 AM records and 355,824 MV records.

The GBIF NZAC dataset had 1186 pairs of duplicate records, in each case with one record with modified date "2016-11-11" and the other with "2017-05-09" (see Results for a likely explanation). I deleted the earlier record versions, reducing the NZAC dataset to 102,092 records.

\section{Issues with field structuring}

Although the ALA and GBIF downloads both contain original and processed data, direct comparisons are not straightforward because of the way the aggregators have structured and filled their data fields. ALA, for example, has duplicated or pseudoduplicated five of its download fields. Simple duplicates are basisOfRecord (two fields) and recordedBy_raw (three fields). dcterms:bibliographicCitation (two fields) is pseudoduplicated, with different entries in the two replicates (noted in another ALA download; the fields are blank in the two downloads audited here). There are two class fields, and ALA explains in the headings.csv file included in the download archive that one class field contains "Class matched / The class the ALA has matched this record to in the NSL [National Species Lists] http://rs.tdwg.org/dwc/terms/class", while the other is only explained as "http://rs.tdwg.org/dwc/terms/class". A check of MV data indicates that the second class field contains original data items, and it is surprising that ALA does not label this field class_raw (as it has done with kingdom_raw, phylum_raw, order_raw, family_raw and genus_raw). The fifth duplicated field is more problematic. ALA generates two specificEpithet fields and one specificEpithet_raw field, with the following explanations in headings.csv:

specificEpithet = "Species matched / Original scientific name supplied with the record

http://rs.tdwg.org/dwc/terms/scientificName" specificEpithet = "http://rs.tdwg.org/dwc/terms/specificEpithet" specificEpithet_raw = "http://rs.tdwg.org/dwc/terms/specificEpithet"

Contradicting the explanations, the first specificEpithet field is not a duplicate of scientificName_raw (also provided in the download), the second specificEpithet holds the originally supplied species name and is therefore actually specificEpithet_raw, and specificEpithet_raw is blank. 
The ALA download also includes the confusingly named:

verbatimDepth = "http://rs.tdwg.org/dwc/terms/verbatimDepth" verbatimDepth_raw = "http://rs.tdwg.org/dwc/terms/verbatimDepth" verbatimElevation = "http://rs.tdwg.org/dwc/terms/verbatimElevation" verbatimElevation_raw = "http://rs.tdwg.org/dwc/terms/verbatimElevation"

The two "raw" fields are empty in both the AM and MV datasets, but in another dataset I examined (National Herbarium of Victoria records, NHV; https://collections.ala.org.au/public/show/co55, accessed 2018-03-14) it is clear that the processing from verbatimElevation_raw to verbatimElevation is intended to convert elevations in units other than metres to elevations in metres. The "raw" entry "985ft", for example, was processed as " 300.228 ". Where verbatimElevation_raw is already in metres in the NHV dataset, verbatimElevation either repeated the entry with added $0.1 \mathrm{~m}$ precision (e.g. "1616" becomes "1616.0") or deleted the entry if it was not simply parseable (e.g. verbatimElevation_raw $=$ " $1627.000 \mathrm{~m}$ ", verbatimElevation blank). ALA processing also failed with range entries, e.g. "15000-17000 ft" was not converted to metres.

GBIF has not duplicated any fields or confused the field naming in its download, but verbatim.txt and occurrence.txt differ significantly in their field structure. The associatedMedia, geodeticDatum, verbatimCoordinates, verbatimLatitude, verbatimLongitude and scientificNameAuthorship fields are dropped without replacement during processing, for unknown reasons. The country field is dropped but its items are processed (with additions, corrections or exclusions) into countryCode in occurrence.txt. Minimum and maximum depth and elevation are recalculated by GBIF during processing. In occurrence.txt, minimumDepthInMeters and maximumDepthInMeters are replaced by depth and depthAccuracy, where "depth" is either the single depth value supplied, or the mean of the supplied minimum and maximum, and "depthAccuracy" is the average deviation from the mean. minimumElevationInMeters and maximumElevationInMeters are similarly replaced by elevation and elevationAccuracy.

GBIF adds genericName and species fields to its processed tables. The terms are defined by GBIF online (http://gbif.github.io/dwc-api/apidocs/org/gbif/dwc/terms/ GbifTerm.html; accessed 2018-02-15) but neither term is part of the Darwin Core standard (see http://rs.tdwg.org/dwc/terms/). The first field is "The genus part of the scientific name", yet in many MV records genericName contains a non-genus name. The species field contains "The canonical name without authorship of the accepted [processed] species" and seems to be the same as the species field in the recommended GBIF download. I ignored the genericName and species fields in the audit.

I also found that there are ALA fields populated with data items with the corresponding GBIF fields completely blank. These are not losses due to processing, since the fields are also blank in the verbatim.txt file. The field contents were evidently not supplied to GBIF, either by ALA, which acts as Australia's GBIF node, or by the data provider. For example, AM catalogNumber C.153619.002 appears on the ALA website 
(https://biocache.ala.org.au/occurrences/4b52f4f9-01e0-411c-adc5-213e40a40b0f; accessed 2018-03-15) and in the download with the habitat entry "On pink Aplysilla" (original and processed), but there is no habitat entry on the corresponding GBIF webpage (https://www.gbif.org/occurrence/1100892212; accessed 2018-03-15), and the habitat field in the GBIF-AM verbatim.txt file is blank. In the AM dataset the blanked fields are acceptedNameUsage, associatedMedia, associatedOccurrences, class, dataGeneralizations, day, geodeticDatum, habitat, identificationRemarks, informationWithheld, month, nameAccordingTo, occurrenceStatus, otherCatalogNumbers, samplingProtocol, taxonConceptID, verbatim CoordinateSystem, verbatimEventDate, verbatimLatitude, verbatimLongitude, verbatim TaxonRank, waterBody and year, and in the MV dataset associatedMedia, class, dataGeneralizations, georeferencedBy, georeferenceProtocol, georeferenceSources, information Withheld, nomenclaturalCode, samplingProtocol, taxonConceptID and waterBody.

In all the fields I audited for processing changes, I found that the original data items ("raw" items in ALA, verbatim.txt items in GBIF) were identical in ALA and GBIF, i.e. there was no "cascading effect" of processing changes from ALA (as GBIF node) to GBIF.

\section{Taxon names}

In examining name changes after processing I ignored changes in taxonomic authorship. Whether attached to names or entered in the scientificNameAuthorship field, authorships are often incomplete or incorrect in original records. As noted above, GBIF drops the scientificNameAuthorship field from processed records, instead adding authorship to some, but not all names in the scientificName field.

I also ignored processing changes in the higher classification of taxa, such as changes in family assignments for genera. Although changes in classification can make records harder to discover in a search of aggregated data, those changes reflect differences in the classification schemes used by data providers and aggregators, and might be regarded as matters of opinion by end-users of aggregated data. However, I used higher-taxon changes as guides when looking for incorrect replacements (see Results).

The search for processing effects on names was further limited to records with genus- or species-group scientificName in the original, and I excluded records in which the original scientificName was informal, e.g. "Idiosepius_n.sp._2". Totals examined were 340,998 records in the AM dataset and 331,480 in the MV dataset.

Both ALA and GBIF attempt to match taxon names with names in reference classifications. GBIF uses a "backbone taxonomy" (https://www.gbif.org/dataset/d7dddbf4-2cf0-4f39-9b2a-bb099caae36c; accessed 18 January 2018) and ALA refers to Australian National Species Lists (https://www.ala.org.au/uncategorised/data-processing/; accessed 18 January 2018). Processing of scientificName could result in no change to the name supplied, or in one or more of the following outcomes, listed below with examples from ALA-processed records. 
deleted. Name has no replacement; processing deletes it.

Jaffaia jaffaensis (Blochmann, 1910) (AM catalogNumber C.100786)

fail-match. Name replaced with incertae sedis, with a name from an unrelated branch of the classification, or with an incorrect name, such as a homonym.

The trichopteran Lasiocephala basalis (Kolenati, 1848) (MV TRI43315) was matched to the plant taxon Drosera sect. Lasiocephala

up-match. Name generalised to one at a level in the taxonomic hierarchy above the supplied or appropriate one.

Oliva parkinsoni Prior, 1975 replaced with Oliva (AM C.100860)

down-match. Name particularised to one at a level in the taxonomic hierarchy below the supplied or appropriate one.

Arrenurus (Arrenurus) replaced with Arrenurus madaraszi (MV H14890107)

swap-match. Name replaced with another at the same rank. For ALA records, this category includes species-level names differing only in subgenus.

Polyphrades brevirostris Lea (MV COL100011) replaced with Essolithna rhombus

subgenus. Subgenus added to or deleted from species or subspecies name; no other major changes (ALA records only).

Vexillum (Costellaria) antonelli (Dohrn, 1861) (AM C.407864) replaced with Vexillum antonellii

amended. Only minor change to name spelling or format.

Hasora discolor mastusia Fruhstorfer, 1911 (MV LEP11) replaced with Hasora discolora mastusia

For each record in which the processed scientificName differed from the original scientificName, I tabulated catalogNumber, original name, processed name, change type (one of the categories listed above), change detail and original typeStatus. An example from ALA:

T4607 | Culex (Lutzia) douglasi Dobrotworsky | Culex (Neoculex) douglasi | swap-match $\mid$ species for species | Holotype

Obviously, a processed scientificName entry may represent more than one kind of change. For example, an up-matched taxon may also be a swap-match at the higher taxon level, as with Anaxo cylindricus obscurus Blackburn (MVT13669) up-matched to the synonym Lepturidea cylindrica by ALA. In the change tables, the ranking order for non-deleted names is fail-match $>$ (up-match $=$ down-match) $>$ swap-match $>$ subgenus $>$ amended. Because GBIF does not usually include subgenera in processed names, the GBIF change tables do not include "subgenus"-type entries, and only a few changes involving original 
subgenera could be included in other categories. It is also likely that at least some of the up-, down- and swap-matched records are actually fail-matched (see Results).

The four name change tables for AM-ALA, AM-GBIF, MV-ALA and MV-GBIF are included in the Zenodo archive with the records downloads.

\section{Results}

\section{Name changes: $A L A$}

Including all change types, ALA changed formal names in the genus- and speciesgroups in 72,963 records in the AM dataset (21.4\%) and 46,835 (14.1\%) in the MV dataset (Table 1). Ignoring the less significant "subgenus" and "amended" change types, the totals are 62,824 (AM, 18.4\%) and 38,374 records (MV, 11.6\%.).

Names deleted in processing are missing from the standard ALA download but still appear on the ALA website. An example is the record for the onychophoran Planipapillus bulgensis Reid (MV K3033) at https://biocache.ala.org.au/occurrences/ e96c0cd8-79ce-43ae-82b4-90f9a2d7d6ac (accessed 28 February 2018). The webpage displays the name and classification for this museum specimen lot, but the "original vs processed values" dialog box shows that the supplied scientificName has been filtered out, and this webpage is not found with a search in ALA for "Planipapillus bulgensis". (GBIF did not delete or change any of the names deleted by ALA.)

The two records down-matched from genus to species (MV HET19158, HET19159) are for specimen lots of the moth Praxis edwardsii. MV supplied the specificEpithet "edwardsii" to ALA but omitted the epithet from scientificName. Some other down-matches are a little surprising as they occur not through replacement by a synonym, but within the same parent taxon. For example, the ant species Pheidole bos has three valid subspecies in Australia (https://biodiversity.org.au/afd/taxa/Pheidole/ names; accessed 28 February 2018). MV records for Pheidole bos baucis (HYM46113) and $P$. bos eubos (HYM46138) have taxonRank originally specified as "subspecies" and are processed without change as subspecies. Five records for $P$. bos with taxonRank specified as "species" and with no subspecific name supplied are down-matched to $P$. bos bos (HYM46132-HYM46136) and re-ranked as "subspecies". The down-matching noted in the Methods section, of Arrenurus (Arrenurus) to Arrenurus madaraszi (MV H14890107), is likewise hard to understand, as MV did not specify a species, the subgenus Arrenurus contains numerous species and $A$. madaraszi is placed in the subgenus Micruacarus. (GBIF did not down-match any of the names down-matched by ALA.)

\section{Name changes: $G B I F$}

Including all change types, GBIF changed formal names in the genus- and speciesgroups in 50,080 records in the AM dataset (14.7\%) and 44,519 (13.4\%) in the MV 
Table I. Tallies of records with changes by ALA in genus- and species-group names in the AM and MV datasets. Totals of records with formal, genus- and species-group names: $\mathrm{AM}=340998, \mathrm{MV}=331480$.

\begin{tabular}{|c|c|c|c|c|c|}
\hline \multicolumn{3}{|c|}{ AM: } & \multicolumn{3}{|c|}{ MV: } \\
\hline deleted & genus & 116 & deleted & genus & 37 \\
\hline deleted & species & 726 & deleted & species & 98 \\
\hline fail-match & genus for plant & 2 & fail-match & species for plant & 1 \\
\hline fail-match & species for plant & 22 & down-match & genus to species & 2 \\
\hline down-match & genus to subgenus & 21 & down-match & genus to subgenus & 727 \\
\hline down-match & species to subspecies & 2041 & down-match & subgenus to species & 2 \\
\hline up-match & genus to class & 134 & down-match & species to subspecies & 1093 \\
\hline up-match & genus to order & 1 & up-match & genus to class & 3 \\
\hline up-match & genus to family & 1317 & up-match & genus to order & 83 \\
\hline up-match & subgenus to family & 6 & up-match & genus to family & 4157 \\
\hline up-match & subgenus to genus & 65 & up-match & genus to subfamily & 79 \\
\hline up-match & species to class & 257 & up-match & subgenus to genus & 93 \\
\hline up-match & species to superfamily & 2 & up-match & species to class & 2 \\
\hline up-match & species to family & 8015 & up-match & species to order & 173 \\
\hline up-match & species to genus & 21641 & up-match & species to family & 1575 \\
\hline up-match & species to subgenus & 40 & up-match & species to subfamily & 159 \\
\hline up-match & subspecies to class & 25 & up-match & species to tribe & 310 \\
\hline up-match & subspecies to family & 244 & up-match & species to genus & 8973 \\
\hline up-match & subspecies to genus & 397 & up-match & species to subgenus & 72 \\
\hline up-match & subspecies to species & 1154 & up-match & subspecies to family & 68 \\
\hline swap-match & genus for genus & 14 & up-match & subspecies to genus & 374 \\
\hline swap-match & species for species & 26462 & up-match & subspecies to subgenus & 22 \\
\hline swap-match & subspecies for subspecies & 122 & up-match & subspecies to species & 6107 \\
\hline subgenus & added to species & 1980 & swap-match & genus for genus & 752 \\
\hline subgenus & deleted from species & 338 & swap-match & subgenus for subgenus & 8 \\
\hline amended & species for species & 7338 & swap-match & species for species & 11438 \\
\hline amended & subgenus for subgenus & 212 & swap-match & subspecies for subspecies & 1966 \\
\hline \multirow[t]{7}{*}{ amended } & subspecies for subspecies & 1 & subgenus & added to species & 3959 \\
\hline & & & subgenus & deleted from species & 2083 \\
\hline & & & subgenus & added to subspecies & 119 \\
\hline & & & subgenus & deleted from subspecies & 134 \\
\hline & & & amended & genus for genus & 3 \\
\hline & & & amended & species for species & 1807 \\
\hline & & & amended & subspecies for subspecies & 356 \\
\hline Total & & 72693 & Total & & 46835 \\
\hline
\end{tabular}

dataset (Table 2). Ignoring the less significant "amended" change type, the totals are 47,453 (AM, 13.9\%) and 37,124 records (MV, 11.2\%.).

GBIF deleted no names in processing. One fail-matched record is for AM catalogNumber C.479173, identified as "Aplacophora" by AM and replaced by GBIF with the bivalve genus Aulacophora Jeffreys, 1882. The other fail-matches are for the marine snail names Nuculana pala (Hedley, 1907) (12 records) and Nuculana (Ledella) pala (Hedley, 
Table 2. Tallies of records with changes by GBIF in genus- and species-group names in the AM and MV datasets. Totals of records with formal, genus- and species-group names: AM $=340998, M V=331480$.

\begin{tabular}{c|c|c|c|c|c}
\hline \multicolumn{2}{c|}{ AM: } & \multicolumn{3}{c}{ MV: } \\
\hline fail-match & class to genus & 1 & swap-match & genus for genus & 3021 \\
\hline fail-match & species for species & 13 & up-match & genus to family & 101 \\
\hline swap-match & species for species & 218 & up-match & species to order & 8 \\
\hline up-match & genus to family & 30 & up-match & species to family & 169 \\
\hline up-match & species to phylum & 2 & up-match & species to genus & 14957 \\
\hline up-match & species to family & 261 & up-match & subspecies to genus & 368 \\
\hline up-match & species to genus & 46900 & up-match & subspecies to species & 18500 \\
\hline up-match & species to subgenus & 5 & amended & genus for genus & 3 \\
\hline up-match & subspecies to genus & 1 & amended & species for species & 5875 \\
\hline up-match & subspecies to species & 22 & amended & subspecies for subspecies & 1517 \\
\hline amended & genus for genus & 53 & Total & & 44519 \\
\hline amended & species for species & 2574 & & & \\
\hline Total & & 50080 & & & \\
\hline
\end{tabular}

Table 3. Tallies of records in which either ALA or GBIF changed formal genus- and species-group names.

\begin{tabular}{c|c|c}
\hline \multicolumn{3}{|c}{ AM (340998 records): } \\
\hline ALA only & ALA and GBIF & GBIF only \\
\hline 56123 & 16570 & 33510 \\
\hline \multicolumn{3}{|c}{ MV (331480 records): } \\
\hline ALA only & ALA and GBIF & GBIF only \\
\hline 36467 & 10368 & 34151 \\
\hline
\end{tabular}

1907) (one record), which were incorrectly matched with Nuculana pella (Linnaeus, 1758) (pala: http://www.marinespecies.org/aphia.php?p=taxdetails\&id=506315, accessed 2018-03-03; pella: http://www.marinespecies.org/aphia.php?p=taxdetails\&id=140578, accessed 2018-03-03). (ALA did not change "Aplacophora" in processing, and swapmatched the pala names to Ledella pala.)

\section{Name changes: $A L A$ vs $G B I F$}

Despite the roughly comparable numbers of name changes, ALA and GBIF processed the same set of names very differently. Table 3 tallies these differences as numbers of records. The overlap (names changed by both ALA and GBIF) is remarkably low. Further, among records with names changed by both ALA and GBIF there was substantial lack of agreement on the type of change (Table 4). However, for most of the records with both ALA and GBIF up-matching the original name, the two processed names were the same, with exceptions generally limited to genus differences (species up-matched to genus) or species differences (subspecies up-matched to species). 
Table 4. Tallies of name change types among records in which both ALA and GBIF changed formal genus- and species-group names.

\begin{tabular}{|c|c|c|}
\hline ALA change type & GBIF change type & No. of records \\
\hline \multicolumn{3}{|l|}{ AM dataset: } \\
\hline deleted & up-match & 34 \\
\hline fail-match & up-match & 1 \\
\hline down-match & up-match & 32 \\
\hline swap-match & swap-match & 4 \\
\hline swap-match & fail-match & 13 \\
\hline swap-match & up-match & 5054 \\
\hline swap-match & amended & 275 \\
\hline up-match & swap-match & 7 \\
\hline up-match & up-match & 8525 \\
\hline up-match & amended & 573 \\
\hline subgenus & up-match & 90 \\
\hline subgenus & amended & 219 \\
\hline amended & up-match & 1275 \\
\hline \multirow[t]{2}{*}{ amended } & amended & 468 \\
\hline & Total & 16570 \\
\hline \multicolumn{3}{|l|}{ MV dataset: } \\
\hline down-match & up-match & 12 \\
\hline down-match & amended & 13 \\
\hline swap-match & swap-match & 8 \\
\hline swap-match & up-match & 700 \\
\hline swap-match & amended & 330 \\
\hline up-match & swap-match & 90 \\
\hline up-match & up-match & 6795 \\
\hline up-match & amended & 1076 \\
\hline subgenus & up-match & 468 \\
\hline subgenus & amended & 38 \\
\hline amended & up-match & 80 \\
\hline \multirow{2}{*}{ amended } & amended & 758 \\
\hline & Total & 10368 \\
\hline
\end{tabular}

In the AM dataset, the four records swap-matched by both ALA and GBIF are for one species:

Gyraulus coranus (Iredale, 1943) (AM)

$>$ Gyraulus (Gyraulus) essingtonensis (ALA)

$>$ Gyraulus corinna (Gray, 1850) (GBIF)

The eight MV records swap-matched by both ALA and GBIF are for two species:

Lipotriches (Hoplonomia) (MV)

$>$ Nomia (Hoplonomia) (ALA)

$>$ Hoplonomia Ashmead, 1904 (GBIF) 
Leioproctus (Nodocolletes) (MV)

$>$ Leioproctus (Lamprocolletes) (ALA)

$>$ Nodocolletes Rayment, 1931 (GBIF)

The 32 AM records with the same species-group name down-matched and upmatched are for:

Erronea chrysostoma Schilder, 1927

$>$ Erronea ovum chrysostoma (ALA)

$>$ Erronea Troschel, 1863 (GBIF)

The 12 MV records with the same name both down- and up-matched are for two species:

Palaminus australiae Fauvel, 1878

> Palaminus australiae australiae (ALA) [ALA here ignores a second subspecies, $P$.

a. hebridensis Cameron, 1934]

> Palaminus Erichson, 1839 (GBIF)

Dabra termitophila Lea, 1906

> Dabra termitophila termitophila (ALA) [ALA here ignores a second subspecies, D. t. victoriensis Lea, 1910]

> Dabra Olliff, 1886 (GBIF)

\section{Name changes: type status}

A consequence of name changes in processing is that a type specimen can lose its association with the name it represents. The AM and MV change tables include numerous records of primary types (Table 5). Among the large number of swap-matches, especially after ALA processing, there are types listed by the aggregator which are not, in fact, types of the replacement name. An example is MVT4295, the holotype of Amaloptila triorbis Turner, 1903, which in ALA has the processed synonym name Elesma subglauca Walker, 1865 with Type status = "holotype" (https://biocache.ala.org.au/occurrences/163727ac8ba3-4dbb-a5c0-bf79d9474f04; accessed 2018-03-03). The holotype of E. subglauca is actually in the Natural History Museum (London) (http://www.nhm.ac.uk/our-science/data/butmoth/search/GenusDetails.dsml?NUMBER=9539.0; accessed 2018-0303). (GBIF did not change "Amaloptila triorbis Turner, 1903".)

When checking for fail-matches (see below), I noted an issue with AM types on the GBIF website. The AM specimen lot C.26622 for Nuculana pala (Hedley, 1907) is a holotype, as can be seen in the "Diagnostics" section of the relevant GBIF webpage (https://www.gbif.org/occurrence/1100962172, accessed 2018-03-03), but although the specimen lot has the processed typeStatus = "holotype" in occurrence.txt, "Type status" is blank on the webpage and the processed value has the remark "Excluded". 
Table 5. Tallies of name change types for primary type specimen lots (holotypes, lectotypes, neotypes, syntypes) among records with formal genus- and species-group names in the AM and MV datasets.

\begin{tabular}{|c|c|c|c|}
\hline \multicolumn{2}{|c|}{ AM-ALA } & \multicolumn{2}{|c|}{ MV-ALA } \\
\hline deleted & 12 & deleted & 27 \\
\hline down-match & 20 & down-match & 28 \\
\hline swap-match & 397 & swap-match & 1637 \\
\hline up-match & 753 & up-match & 560 \\
\hline subgenus & 16 & subgenus & 320 \\
\hline amended & 57 & amended & 46 \\
\hline Total & 1255 & Total & 2618 \\
\hline \multicolumn{2}{|c|}{ AM-GBIF } & \multicolumn{2}{|c|}{ MV-GBIF } \\
\hline fail-match & 1 & swap-match & 3 \\
\hline swap-match & 1 & up-match & 223 \\
\hline up-match & 1326 & amended & 100 \\
\hline amended & 48 & Total & 326 \\
\hline Total & 1376 & & \\
\hline
\end{tabular}

\section{Name changes: unrecognised fail-matches}

Without checking thousands of name changes individually, it was impossible to determine how many up-, down- and swap-matches resulted in a taxon name being replaced with one from another branch of biological classification, or with a non-synonym (see the GBIF palal pella example, above). The 39 fail-matched records tallied in the change tables are the most obvious failures I found. I suspect there are many more, but using higher classifications in name-changed records as a guide was made impractical by unfilled higher-taxon entries $(\mathrm{AM})$ and disagreements on higher taxa between data provider and aggregator (MV).

\section{Data losses}

Aggregator processing sometimes results in loss of a data item: an original record contains a data item in a particular field, but after processing that field is blank for the record concerned. I found a surprisingly high number of data losses in the audited datasets. Below I give examples of loss (see also the comment above on deleted taxon names in ALA, and on verbatim depth and elevation data in Methods: Issues with field structuring). For more details, see data_notes.txt in the Zenodo archive for this project.

identifiedBy: AM-ALA and MV-ALA, 100\% loss in processing. The original identifiedBy_raw data item appears on the ALA webpage as "Identified by" for the record but is missing from the standard (recommended) download).

locality: MV-ALA, $100 \%$ loss in processing. The original locality_raw data item appears on the ALA webpage as "Locality" for the record but is missing from the standard (recommended) download). (locality_raw is blank in the AM dataset.) 
Losses of date information were common and evidently due to processing rules written to deal with various date formats. In the modified field in the NZAC dataset, for example, GBIF successfully parsed 4765 entries in YYYYMM-DDTHH:MM:SS+12:00 format, but deleted 97,327 entries in YYYY-MMDDTHH:MM:SS.sss+12:00 format (95\% data loss). This failure may explain why GBIF did not delete the earlier versions of the 1186 duplicated records (see Methods), as both the earlier and later versions of these records have modified entries in YYYYMM-DDTHH:MM:SS.sss+12:00 format.

Other major losses were in the eventDate and dateIdentified fields and were sometimes inconsistent. Here are details of an example: the MV-ALA dataset contains 341,693 correctly formed entries in eventDate_raw. These include 13,815 interval dates. The entire interval date was excluded when the format was YYYY-MM-DD/ YYYY (23 records) or YYYY-MM-DD/YYYY-MM (32). The earlier date in the interval (only) was accepted from the formats YYYY-M-D/D (1 record), YYYY-MMDD/DD (11037 plus an exception, see below), YYYY-MM-DD/MM-DD (2317) and YYYY-MM-DD/YYYY-MM-DD (404 plus an exception). One of the two exceptional exclusions was the entry "2006-09-02/2005-11-20", which is malformed as an interval date. Its exclusion suggests that ALA tested interval dates before deleting the later date. The second exclusion was "1943-06-20/21" for catalogNumber C.95257 (https:// biocache.ala.org.au/occurrences/5966a91c-b333-4781-924f-92f1f6f57919; accessed 2018-02-21). The same interval date ("1943-06-20/21") was accepted for four other records, e.g. C.95256 (https://biocache.ala.org.au/occurrences/b78583d0-4195-4a65a733-6445394e7bd2; accessed 2018-02-21). Among non-interval dates, ALA excluded YYYY (98812 records) and YYYY-MM (51412), while accepting YYYY-M-D (2), YYYY-MM-D (1) and YYYY-M-DD (1). All but 258 of 177,650 valid YYYY-MMDD dates were accepted. All 258 appear to be well-formed, and as with "1943-0620/21" above, ALA accepted and excluded the same YYYY-MM-DD in different records. For example, "1985-10-06" was rejected in C.364665 (https://biocache.ala.org. au/occurrences/16a2f2ee-a330-4a4a-8a89-b1450f00f270; accessed 2018-02-21) but accepted in C. 441797 (https://biocache.ala.org.au/occurrences/dcdc015f-363d-4d5ea532-2a8a4988ee20; accessed 2018-02-21). In summary, ALA filtered out 150,537 of all valid eventDate_raw entries in the MV dataset (44\% loss) and accepted only the starting date in non-excluded interval dates.

ALA also had processing losses in fields containing names of persons and organisations. In the AM dataset, the original recordedBy_raw field has 236,855 entries in a range of formats. These include both "name" (e.g. "A.C. \& J.E.Miller") and "reverse name" (e.g. "Abbott, E.") entries, as well as oddities such as "aborigines" and "Unknown (Sea Gypsies)". ALA excluded no recordedBy_raw entries and processed 66,900 entries without change. Many of the 169,955 entries changed by ALA ( $72 \%$ of the total) were not processed successfully. Some systematic failures are listed below. The most significant errors were the replacement of a valid string by "null", which occurred in 7,596 entries ( $3.2 \%$ of total), and the data losses associated with conjunction failure (see below), which I did not tally. 
null A name string was replaced by the word "null", e.g. "A.Musgrave \& E.LeG. Troughton" processed as "Musgrave, A.|null".

conjunction failure Conjunctions and separators were replaced with pipes (“|”). The results were variably successful, with some pipes separating two recorders correctly, e.g. "J.\& D.Freeman" processed as "Freeman, J.|Freeman, D.” A large number (not tallied) of entries were incorrectly piped, e.g. "J.Brazier \& G.Rossiter" processed as "Rossiter, J.|Rossiter, G." and "J.Paxton \& M.McGrouther" processed as "Mc, J.|Mc, M.”. Conjunction failures also saw institutional affiliations become separate recorders, as with "Kessner, Mr. Vince - Australian Museum - Malacology" processed as "Kessner, V. Vince|Australian Museum|Malacology”.

initial reversal The order of the first name and middle initial were reversed, e.g. "Harvey, Michael S." processed as "Harvey, S. Michael”.

added initial The initial of the first name is added, e.g. "Houghton, Noel" processed as "Houghton, N. Noel".

initial comma A comma was placed at the beginning of the name(s), e.g. "B.M.R." processed as “, B.M.R.” and "W.F.\& J.M.Ponder \& T.Habe" as “, W.F.|Ponder, J.M.|Habe, T.”.

surname ending The string "and" at the end of a surname was processed as the conjunction "and", e.g. "M. Crossland" replaced by ", M. Crossl|".

GBIF processed most names without change in the recordedBy and identifiedBy fields, but excluded 443 recordedBy entries representing 119 unique name strings in the MV dataset. A check of several of the excluded entries shows that they were accepted by GBIF in other records. For example, "Peter K. Lillywhite - Museum Victoria" was excluded in four records but accepted in 233 others. This inconsistent processing resulted in a loss of $<1 \%$ of valid data items.

\section{Other data issues}

While checking for taxon name changes and data losses, I incidentally noted several other unwelcome results of data processing, described here.

In the NZAC dataset, verbatim.txt has the string "not identified on slide" in the scientificName field for catalogNumber NZAC02015964, and the other taxonomic fields are empty. GBIF matched this string with the fictitious genus Not Chan, 2016, to which occurrence records from other datasets had also been matched, for entries such as "Not naturalised in SA sp." and "Not listed". I published this processing error in a post to the iPhylo blog on 24 January 2018 ( http://iphylo.blogspot.com.au/2018/01/guest- 
post-not-problem.html). The "Not Chan, 2016" page was subsequently removed from the GBIF website and the NZAC "not identified on slide" record is now processed as "incertae sedis" (https://www.gbif.org/occurrence/1315606681; accessed 2018-03-01).

In the MV dataset, ALA processed geodeticDatum_raw entries to geodeticDatum inconsistently and with errors. Of the "WGS84" entries, 341539 were correctly processed to the equivalent EPSG:4326, while 652 were deleted. Also processed to EPSG:4326 were 203 "AGD66" entries, for which the correct equivalent is EPSG:6202; the difference on the ground is ca $200 \mathrm{~m}$.

ALA processes locality text fields on the basis of the supplied decimal latitude and longitude values, disregarding original values in text fields. If the supplied coordinates are incorrect, ALA's processing sometimes adds incorrect text data. In the AM dataset, catalogNumber C.500744 is from Roma Gorge in Australia's Northern Territory but was assigned to Mozambique (“-23.63805 32.41804” supplied instead of -23.63805 132.41804) and C.429548.002 from near Newcastle Waters in the Northern Territory was assigned to Namibia (“-17.25750 13.45444” instead of -17.25750 133.45444). In both cases country_raw is "Australia" and stateProvince_raw is "Northern Territory". In the MV dataset, ALA assigned Coral Bay (country_raw = "Australia", stateProvince_ raw = "Western Australia") to Botswana ("-23.14 23.14" supplied instead of -23.14 113.77; catalogNumber HET5323), and Clifton Downs Station ("Australia" and "New South Wales”) to South Africa (“-29.61 29.61” instead of -29.61 142.57; HET4586).

\section{Discussion}

Users can download from ALA and GBIF, as I did, sets of occurrence records containing both original and processed data items. However, both ALA and GBIF recommend smaller downloads containing only processed records. As noted above, these processed records can have blanks where data was originally provided, can feature taxon names different from the ones used by the data provider, and can contain processing errors. The user of a recommended ALA or GBIF download has no way of knowing which names have been changed or which data items lost, other than to go to the ALA and GBIF websites and look for original vs processed data differences for individual records, or investigate individual data quality "flags" and "assertions".

I do not know the extent to which data providers are informed by aggregators about changes made in the data supplied, or whether providers, under the terms of legal arrangements with aggregators, can ask that changes be reversed. Museums and herbaria might be particularly concerned at the confusion in processed records regarding the identity of type specimens. These questions were addressed in general terms in a published response to an earlier audit of mine which looked at the quality of occurrence data as provided to aggregators (Mesibov 2013):

"Some data providers encourage the ALA to make corrections to the provider's records (for provider and ALA). Other data providers would withdraw their support if similar changes were attempted on their data by the ALA. Feedback from the ALA to 
a data provider may result in immediate corrections (and data propagation) while in other cases, the provider has no resources to resolve an issue. There is no single process here that will work effectively in all circumstances." (Belbin et al. 2013).

Note, however, that these comments referred to problems in data as provided, not to problems generated at aggregator level, as reported here.

It is a little surprising that GBIF asks users to cite their downloaded data as authored by the provider (See Methods, Data sources), and that ALA likewise asks (in each download's citation.csv file) that data be cited as records from the provider. Clearly this is not the case for processed data. It would be more correct to say that aggregated data are made available as the combined work of provider and aggregator, and that the aggregator is solely responsible for any differences between original and processed data.

Some of the processing problems noted in this paper are the result of programming errors. Given the results reported here, it seems unlikely that ALA and GBIF programming staff or contractors have systematically compared original and processed data to look for problems in selected fields, as I did. I am also aware that issues raised on the ALA GitHub site (https://github.com/AtlasOfLivingAustralia) by staff and users can remain open for long periods (e.g. https://github.com/AtlasOfLivingAustralia/ala-downloads/issues/17; accessed 2018-03-03), and that closed issues may still be open, e.g. the lack of a processed locality field, which was said to have been corrected two months before I downloaded ALA data for audit (https:/github.com/AtlasOfLivingAustralia/ ala-downloads/issues/14; accessed 2018-03-03). The apparent failure of ALA and GBIF to monitor processing output is both surprising and disappointing. It is particularly surprising that data loss in processing of dates remains a significant problem, five years after a careful analysis of GBIF date losses was published by Otegui et al. (2013a).

Data loss may be the result of programming "bugs", but both ALA and GBIF regard the replacement of originally supplied taxon names as a feature of their aggregation protocols. This view has its critics (Franz and Sterner 2018), and it is hard to understand how losing a taxon name through fail-matching or up-matching improves an occurrence record. Otegui et al. (2013b) attribute some failed matching to inadequate provision of higher-taxon information in original records. The remarkable lack of agreement between ALA and GBIF on replacement names for the same original names highlights another problem: reference classifications differ. The Catalogue of Life Plus project (https:/github.com/Sp2000/colplus; accessed 2018-03-01) will attempt to replace differing reference classifications with a single "consensus" one for aggregator use, but it seems unlikely that the result will be universally adopted by data providers, let alone by the taxonomists who supply scientific names to museums and herbaria.

Three recommendations could be made to improve the taxonomic usefulness of aggregated occurrence records. A simple, easily implemented one is for aggregators to include both original and processed taxonomic data items in each record, from scientificName and all higher taxon fields. A second improvement would be for aggregators to employ, as Franz and Sterner (2018) propose, multiple reference classifications, so that each original taxon name could be seen in a range of taxonomic contexts. A third possible advance would be for aggregators to construct sets of related name strings, so 
that each original name can be seen by the user as one element in a group of variants, synonyms and fuzzy matches - a "see also" function in searches and record selection for download.

There have been a number of recent studies which question the quality of aggregated occurrence records (see references in Franz and Sterner 2018), but Sikes et al. (2016) defend GBIF and other aggregators by saying that all data need vetting and processing and that the quality of aggregated data is the collective responsibility of the biodiversity data community. These are truisms. It is also obvious that aggregators should not lose or confuse the data they are provided with, and the present audit suggests that both ALA and GBIF could do better. End-users of aggregated occurrence records would be wise to download both the original and the processed datasets, and to check carefully for data losses and taxon name replacements.

\section{Acknowledgements}

I thank reviewers Sarah Faulwetter, Nico Franz and Donald Hobern for very helpful comments on a draft of this paper.

\section{References}

Belbin L, Daly J, Hirsch T, Hobern D, LaSalle J (2013) A specialist's audit of aggregated occurrence records: An 'aggregator's' perspective. ZooKeys 305: 67-76. https://doi.org/10.3897/ zookeys.305.5438

Franz NM, Sterner BW (2018) To increase trust, change the social design behind aggregated biodiversity data. Database 2018: bax100. https://doi.org/10.1093/database/bax100

Mesibov R (2013) A specialist's audit of aggregated occurrence records. ZooKeys 293: 1-18. https://doi.org/10.3897/zookeys.293.5111

Otegui J, Ariño AH, Chavan V, Gaiji S (2013a) On the dates of GBIF mobilised primary biodiversity records. Biodiversity Informatics 8: 173-184. https://journals.ku.edu/index.php/ jbi/article/view/41250, https://doi.org/10.17161/bi.v8i2.4125

Otegui J, Arińo AH, Encinas MA, Pando F (2013b) Assessing the primary data hosted by the Spanish node of the Global Biodiversity Information Facility (GBIF). PLoS ONE 8(1): e55144. https://doi.org/10.1371/journal.pone.0055144

Robbins AD (2018) GAWK: Effective AWK programming. A user's guide for GNU Awk, edition 4.2. Free Software Foundation, Boston. https:/www.gnu.org/software/gawk/manual/gawk.pdf

Sikes DS, Copas K, Hirsch T, Longino JT, Schigel D (2016) On natural history collections, digitized and not: a response to Ferro and Flick. ZooKeys 618: 145-158. https://doi.org/10.3897/ zookeys.618.9986

Wieczorek J, Bloom D, Guralnick R, Blum S, Döring M, Giovanni R, Robertson T, Vieglais D (2012) Darwin Core: an evolving community-developed biodiversity data standard. PLoS ONE 7(1): e29715. https://doi.org/10.1371/journal.pone.0029715 